\title{
Examining the Theoretical Framework of Behavioral Activation for Major Depressive Disorder: Smartphone-Based Ecological Momentary Assessment Study
}

Claire Rosalie van Genugten ${ }^{1,2,3}$, MSc; Josien Schuurmans ${ }^{1,2}, \mathrm{PhD}$; Adriaan W Hoogendoorn ${ }^{1,2}, \mathrm{PhD}$; Ricardo Araya ${ }^{4}$, $\mathrm{PhD}$; Gerhard Andersson ${ }^{5,6}, \mathrm{PhD}$; Rosa Baños ${ }^{7,8,9}$, PhD; Cristina Botella ${ }^{8,10}, \mathrm{PhD}$; Arlinda Cerga Pashoja ${ }^{11}$, PhD; Roman Cieslak $^{12,13}$, PhD; David Daniel Ebert ${ }^{14}$, PhD; Azucena García-Palacios ${ }^{8,10}$, PhD; Jean-Baptiste Hazo ${ }^{15,16}$, MSc; Rocío Herrero $^{7,8}$, PhD; Jérôme Holtzmann ${ }^{17}$, PhD; Lise Kemmeren ${ }^{1,2}$, MSc; Annet Kleiboer ${ }^{3}$, PhD; Tobias Krieger ${ }^{18}$, $\mathrm{PhD}$; Ewelina Smoktunowicz ${ }^{12}, \mathrm{PhD}$; Ingrid Titzler ${ }^{19}, \mathrm{MSc}$; Naira Topooco ${ }^{5,20}, \mathrm{PhD}$; Antoine Urech ${ }^{18}, \mathrm{PhD}$; Johannes H Smit $^{1,2}$, PhD; Heleen Riper ${ }^{1,2,3,21,22}$, PhD

${ }^{1}$ Department of Research and Innovation, GGZ inGeest, Specialized Mental Health Care, Amsterdam, Netherlands

${ }^{2}$ Department of Psychiatry, Amsterdam Public Health Institute, Amsterdam University Medical Center, Vrije Universiteit, Amsterdam, Netherlands

${ }^{3}$ Department of Clinical, Neuro and Developmental Psychology, Vrije Universiteit, Amsterdam, Netherlands

${ }^{4}$ Institute of Psychiatry Psychology and Neurosciences, King's College London, London, United Kingdom

${ }^{5}$ Department of Behavioural Sciences and Learning, Linköping University, Linköping, Sweden

${ }^{6}$ Centre for Psychiatry Research, Department of Clinical Neuroscience, Karolinska Institutet, Stockholm, Sweden

${ }^{7}$ Polibienestar Research Institute, University of Valencia, Valencia, Spain

${ }^{8}$ Institute of Health Carlos III, CIBERObn CB06 03/0052, Madrid, Spain

${ }^{9}$ Department of Personality, Evaluation and Psychological Treatment, Faculty of Psychology, University of Valencia, Valencia, Spain

${ }^{10}$ Department of Basic and Clinical Psychology and Psychobiology, Faculty of Health Sciences, Jaume I University, Castellon de la Plana, Spain

${ }^{11}$ Department of Population Health, London School of Hygiene \& Tropical Medicine, London, United Kingdom

${ }^{12}$ Faculty of Psychology, SWPS University of Social Sciences and Humanities, Warsaw, Poland

${ }^{13}$ Lyda Hill Institute for Human Resilience, Colorado Springs, Colorado Springs, CO, United States

${ }^{14}$ Department for Sport and Health Sciences, Technical University Munich, Munich, Germany

${ }^{15}$ Eceve, Unit 1123, Inserm, University of Paris, Paris, France

${ }^{16}$ Health Economics Research Unit, Assistance Publique-Hôpitaux de Paris, Paris, France

${ }^{17}$ Mood Disorders and Emotional Pathologies Unit, Pôle de Psychiatrie, Neurologie et Rééducation Neurologique, University Hospital Grenoble Alpes, Grenoble, France

${ }^{18}$ Department of Clinical Psychology, University of Bern, Bern, Switzerland

${ }^{19}$ Department of Clinical Psychology and Psychotherapy, Institute of Psychology, Friedrich-Alexander-University Erlangen-Nürnberg, Erlangen, Germany

${ }^{20}$ Centre for m2health, Palo Alto University, Palo Alto, CA, United States

${ }^{21}$ Institute of Telepsychiatry, University of Southern Denmark, Odense, Denmark

${ }^{22}$ Faculty of Medicine, University of Turku, Turku, Finland

\section{Corresponding Author:}

Claire Rosalie van Genugten, MSc

Department of Research and Innovation

GGZ inGeest

Specialized Mental Health Care

Oldenaller 1

Amsterdam, 1081HJ

Netherlands

Phone: 310207884666

Email: c.genugten@ggzingeest.nl

\section{Abstract}


Background: Behavioral activation (BA), either as a stand-alone treatment or as part of cognitive behavioral therapy, has been shown to be effective for treating depression. The theoretical underpinnings of BA derive from Lewinsohn et al's theory of depression. The central premise of BA is that having patients engage in more pleasant activities leads to them experiencing more pleasure and elevates their mood, which, in turn, leads to further (behavioral) activation. However, there is a dearth of empirical evidence about the theoretical framework of BA.

Objective: This study aims to examine the assumed (temporal) associations of the 3 constructs in the theoretical framework of BA.

Methods: Data were collected as part of the "European Comparative Effectiveness Research on Internet-based Depression Treatment versus treatment-as-usual" trial among patients who were randomly assigned to receive blended cognitive behavioral therapy (bCBT). As part of bCBT, patients completed weekly assessments of their level of engagement in pleasant activities, the pleasure they experienced as a result of these activities, and their mood over the course of the treatment using a smartphone-based ecological momentary assessment (EMA) application. Longitudinal cross-lagged and cross-sectional associations of 240 patients were examined using random intercept cross-lagged panel models.

Results: The analyses did not reveal any statistically significant cross-lagged coefficients (all $P>.05$ ). Statistically significant cross-sectional positive associations between activities, pleasure, and mood levels were identified. Moreover, the levels of engagement in activities, pleasure, and mood slightly increased over the duration of the treatment. In addition, mood seemed to carry over, over time, while both levels of engagement in activities and pleasurable experiences did not.

Conclusions: The results were partially in accordance with the theoretical framework of BA, insofar as the analyses revealed cross-sectional relationships between levels of engagement in activities, pleasurable experiences deriving from these activities, and enhanced mood. However, given that no statistically significant temporal relationships were revealed, no conclusions could be drawn about potential causality. A shorter measurement interval (eg, daily rather than weekly EMA reports) might be more attuned to detecting potential underlying temporal pathways. Future research should use an EMA methodology to further investigate temporal associations, based on theory and how treatments are presented to patients.

Trial Registration: ClinicalTrials.gov, NCT02542891, https://clinicaltrials.gov/ct2/show/NCT02542891; German Clinical Trials Register, DRKS00006866, https://tinyurl.com/ybja3xz7; Netherlands Trials Register, NTR4962, https://www.trialregister.nl/trial/4838; ClinicalTrials.Gov, NCT02389660, https://clinicaltrials.gov/ct2/show/NCT02389660; ClinicalTrials.gov, NCT02361684, https://clinicaltrials.gov/ct2/show/NCT02361684; ClinicalTrials.gov, NCT02449447, https://clinicaltrials.gov/ct2/show/NCT02449447; ClinicalTrials.gov, NCT02410616, https://clinicaltrials.gov/ct2/show/NCT02410616; ISRCTN registry, ISRCTN12388725, https://www.isrctn.com/ISRCTN12388725

(JMIR Ment Health 2021;8(12):e32007) doi: 10.2196/32007

\section{KEYWORDS}

depression; behavioral activation; theoretical framework; ecological momentary assessment; random-intercept cross-lagged panel model; behavior; framework; EMA; smartphone; mental health; treatment; engagement; mood

\section{Introduction}

Most psychotherapeutic treatments for depression are underpinned by a clear theoretical framework of how a specific therapy is supposed to engender change in patients' mood states. In light of the fact that various psychotherapies have been proven to be effective for treating depression (eg, [1-4]), one would perhaps assume that theoretical frameworks are also evidence based. However, demonstrating that a therapy is effective is not the same as providing evidence or explanations as to how it actually works [5-8], that is, the process through which a certain variable leads to change in another variable $[6,7]$. In addition to effectiveness studies that target an overall treatment package, understanding how therapy targets the interplay between specific factors that are believed to be of importance can ultimately give rise to specific treatment recommendations or improvements to treatment protocols (eg,
[6-10]). Conducting a verification of the underlying theoretical framework of a form of psychotherapy can help optimize treatment strategies (eg, [6-10]), that is, direct better, stronger, or different treatment strategies that underpin the critical processes of the treatment (eg, [6-10]).

One such effective psychotherapeutic intervention for depression is behavioral activation (BA). The empirical evidence for BA is both extensive and convincing; it can be offered either as a stand-alone treatment or within the setting of cognitive behavioral therapy (CBT) [11-13]. BA is based on Lewinsohn et al's $[14,15]$ theory of depression, which purports that when a person is depressed, they tend to engage less in pleasant or meaningful activities, resulting in them experiencing less pleasure, which, in turn, leads to an increased depressed mood, and so on. From this perspective, persons end up in a vicious circle of depression [14,15], as depicted in the left panel of Figure 1. 
Figure 1. Circles of depression and BA based on the theoretical framework of depression by Lewinsohn et al [14,15]. BA: behavioral activation.

Circle of depression

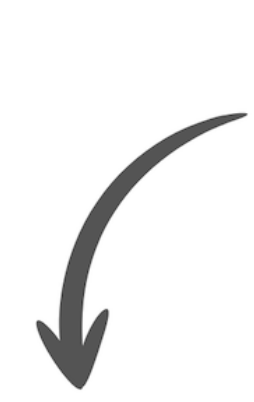

Increased

depressed $\operatorname{mood}$

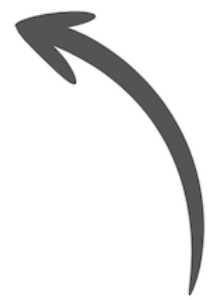

Not engage in

pleasant

activities
Experience

less

pleasure

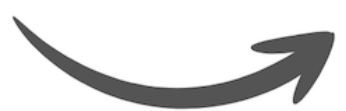

Through BA, patients learn from self-monitoring their everyday activities and related mood that the way they behave affects the way they feel, while conversely, the way they feel affects the way they behave $[11,12,16]$. Therapists motivate their patients to schedule and engage in everyday pleasant activities [12,17]. Patients are informed that engaging in these activities may not lead them to immediately experience greater pleasure and that experiencing pleasure may not enhance their mood straightaway. Rather, they are told that this is a process that requires time and that positive results will follow in due course as they continue to engage in pleasurable activities $[12,17]$. The circle of BA, as it is referred to in Lewinsohn et al's $[14,15]$ model is shown in the right panel of Figure 1. As one can see in Figure 1, BA aims to break the vicious circle of depression by increasing the patient's engagement in pleasant activities.

Currently, there is a relative dearth of empirical evidence available concerning the assumed causal pathways in the theoretical framework of BA [10,18-20]. Janssen et al [20] sought to address this gap in extant knowledge by conducting a systematic review in which they investigated the mediators of BA for depression. In this review [20], the evidence for the mediating role played by both engaging in pleasant activities and experiencing pleasure was not consistently replicated across the considered studies. However, the authors did conclude that their review was not without its limitations. First, some of the included studies were of poor methodological quality, while different questionnaires were used to assess the mediators. Moreover, the results of the mediational analysis did not necessarily explain the processes via which change occurs, insofar as most studies solely focused on unidirectional relationships, in addition to lacking temporal dependency $[9,20]$. It is thought that the reciprocal interactions and longitudinal associations between different variables determine psychological functioning [21]. Thus, to successfully delineate the theoretical framework of BA, further research is needed that considers reciprocal interactions and longitudinal associations by using ecological momentary assessment (EMA) methods $[8,10,20]$.

\section{$\underline{\text { Circle of behavioral activation }}$}

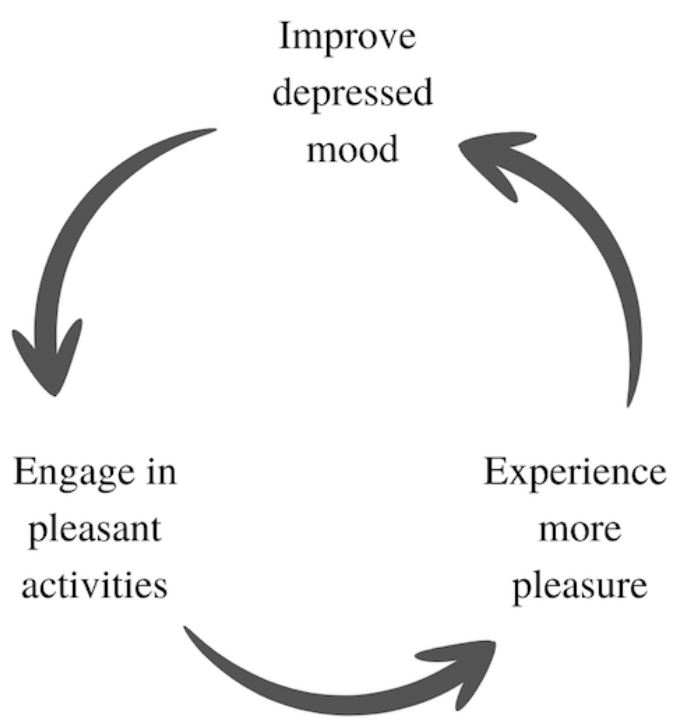

EMA methods allow us to trace temporal pathways of change across different variables among patients during the course of treatment $[9,10,20,22]$. With EMA methods, or real-time monitoring, persons are routinely asked to report on their mood and other related phenomena while they are in their own ecological habitat (eg, [23-25]). Traditionally, EMA was conducted via paper-and-pencil diaries as well as via stand-alone technical devices $[25,26]$. Today, EMA is often facilitated by smartphone-based applications $[25,27,28]$. The fact that phenomena are measured close to their occurrence helps to avoid recall bias as much as possible $[29,30]$. This fact is especially pertinent with respect to patients suffering from mood disorders, as prior research has shown that recall among this patient group is confounded by current feelings, not to mention greater recall of unpleasant activities than pleasant activities [31-33]. The past several decades have seen a surge in the use of EMA methods in mood disorder research.

This paper aims to examine the assumed (temporal) associations of the 3 constructs in the theoretical framework of BA among major depressive disorder (MDD) patients, who monitored their engagement in pleasant activities, the pleasure they experienced as a result of conducting these activities, and their related mood states using smartphone-based EMA during the course of blended CBT (bCBT) in routine mental health care (MHC). bCBT integrates face-to-face (f-t-f) sessions with both weband smartphone-based components into 1 treatment protocol [34]. Data from a subsample of the patients receiving bCBT and smartphone-based EMA $(\mathrm{N}=240)$ as part of the European Comparative Effectiveness Research on Internet-based Depression Treatment treatment-as-usual (TAU) (E-COMPARED) trial were used to investigate the longitudinal reciprocal associations [35,36]. 


\section{Methods}

\section{Participants, Procedure, and Treatment Protocol}

E-COMPARED is a randomized controlled, non-inferiority trial that was conducted across 8 European countries. The principal aim of the trial was to compare both the clinical effectiveness and cost-effectiveness of bCBT to treatment-as-usual (TAU) for MDD. For the trial, a generic bCBT protocol was established $[35,36]$. The study protocol of E-COMPARED gives a complete overview of the bCBT protocol [36]. In short, the core components of the bCBT protocol mirrored traditional f-t-f CBT: (1) psychoeducation, (2) BA, (3) cognitive restructuring, and (4) relapse prevention. However, with bCBT, f-t-f sessions were replaced by and alternated with online modules [34,37]. The themes of the f-t-f sessions matched the content of the online sessions. The more practical components were delivered online (ie, psychoeducation, completing homework assignments). The focus of the health care professional during the $\mathrm{f}-\mathrm{t}-\mathrm{f}$ session was on process-related treatment outcomes (ie, discussion of feelings and thoughts), discussing homework assignments, and providing support [36]. Although the protocols included the same core components (including BA), bCBT was delivered in the primary care of specialized MHC and therapists were granted some freedom to customize the protocol to their local needs. To make sure the protocol suited their local context, the therapists were allowed to adapt the treatment duration and the ratio between the number of $\mathrm{f}-\mathrm{t}-\mathrm{f}$ sessions and online sessions. In addition, therapists were allowed to include some additional components (eg, mindfulness or problem solving), but this could not take up more than $25 \%$ of the total treatment (f-t-f and online sessions combined). Moreover, based on local availability, different web- and smartphone-based applications were used. Table 1 shows the bCBT format per country.

Table 1. Blended cognitive behavioral therapy format per country.

\begin{tabular}{|c|c|c|c|c|c|c|}
\hline Country & Type of care & $\begin{array}{l}\text { Treatment duration } \\
\text { (weeks) }\end{array}$ & Face-to-face sessions, $\mathrm{n}$ & Web-based modules, n & $\begin{array}{l}\text { Total number of } \\
\text { sessions, } n\end{array}$ & Sequencing \\
\hline Germany & Primary & $11-13$ & 6 & 10 & 16 & Alternate \\
\hline Poland & Primary & $6-10$ & 7 & 6 & 13 & Alternate \\
\hline England & Primary & 11 & 5 & 6 & 11 & Alternate \\
\hline Spain & Primary & 10 & 10 & 10 & 20 & Alternate \\
\hline Sweden & Primary & 10 & 4 & 6 & 10 & Alternate \\
\hline The Netherlands & Specialized & $18-20$ & 10 & 9 & 19 & Alternate \\
\hline France & Specialized & $16-20$ & 8 & 8 & 16 & Alternate \\
\hline Switzerland & Specialized & 18 & 9 & 9 & 18 & Alternate \\
\hline
\end{tabular}

As with traditional $\mathrm{f}-\mathrm{t}-\mathrm{f} \mathrm{CBT}$, the BA component in this study was based on Lewinsohn et al's [14,15] theory of depression and aimed at increasing patients' engagement in activities. The BA component started early in treatment and remained a recurring topic in the (f-t-f) sessions throughout the course of treatment. During the f-t-f sessions, the patient was motivated by the health care professional to schedule and engage in (potential) pleasant activities. The patient could (re)read the rationale of BA online, read a so-called activity-list that could be used for inspiration, and use the platform as a tool to specify and schedule which activities to engage in that week. A smartphone-based EMA application was used to monitor engagement in activities, pleasure experienced as a result of these activities, and the mood state over the course of treatment. Although the f-t-f and online sessions were only approximately (alternated) once a week, the patient was encouraged to take an active role in their therapy and to practice in their own environment in between the sessions.

Between February 2015 and December 31, 2017, patients were recruited from primary care (Germany, Poland, Spain, Sweden, and the United Kingdom) and outpatient departments of specialized MHC settings (France, the Netherlands, and Switzerland) $[35,36]$. Patients were asked by their health care professionals whether they were willing to take part in the study. The inclusion criteria were as follows: (1) must be at least 18 years of age; (2) meet the Diagnostic and Statistical Manual of
Mental Disorders, Fourth Edition (DSM-IV), criteria for MDD, as confirmed by Mini International Neuropsychiatric Interview (M.I.N.I.) version 5.0 [38,39]; and (3) report mild-to-severe depressive symptoms (score of $\geq 5$ ) on the Patient Health Questionnaire-9 (PHQ-9) [40,41]. The exclusion criteria were as follows: (1) already receiving psychological treatment for depression in a primary or specialized MHC setting; (2) be at high risk for suicide or have a DSM-IV diagnosis of substance dependence, bipolar disorder, psychotic illness, or obsessive compulsive disorder, as confirmed by M.I.N.I. version 5.0 [38,39]; (3) not able to comprehend the spoken and written language of their country of residence; (4) not have access to a computer with a fast internet connection; and (5) not have a smartphone compatible with the Android operating system or be unwilling to carry a smartphone provided by the research team. For more in-depth information about the specifics of both country and setting recruitment procedures, please see elsewhere [35,36].

Patients who met the inclusion criteria $(\mathrm{N}=943)$ were randomly allocated to receive either bCBT $(n=476)$ or TAU $(n=467)$. For the purposes of this study, only patients who were randomized to receive bCBT were initially selected, since patients who were allocated to the TAU group were not invited to complete the smartphone-based EMA measures. Of the 476 bCBT patients, 152 did not receive treatment (never attended the first f-t-f session, dropped out after the first f-t-f session, never logged 
onto the platform) or did not provide any weekly EMA reports. Of the remaining 324 patients, all patients reported on their mood, but 84 of them failed to complete reports on their levels of activities or pleasure. This led to an analytic sample of 240 patients who reported on all 3 variables of interest (activities, pleasure, and mood) in the weekly EMA reports. Potential selection bias was examined by analyzing potential differences in terms of demographic and clinical characteristics between the E-COMPARED patients who were randomly allocated to receive bCBT but did not meet the study's inclusion criteria (n $=476-240=226$ ) and patients who were included in this study $(\mathrm{N}=240)$. The results of these analyses are presented in the Result section.

\section{Measures}

\section{Demographic and Clinical Characteristics}

At baseline, information about demographic and clinical characteristics was gathered. The basic demographics included age, gender, and educational level. This information was obtained through a web-based questionnaire. The clinical characteristics included current MDD diagnosis and other (comorbid) psychiatric diagnoses, severity of depression, and use of antidepressant medication. The presence of current MDD and current comorbid psychiatric disorders (dysthymia, panic disorder with or without agoraphobia, agoraphobia, social phobia, generalized anxiety disorder, posttraumatic stress disorder) was defined according to DSM-IV criteria [39] and established using M.I.N.I. version 5.0 [38]. Patients reported on the severity of their depression by completing a web-based version of the PHQ-9 [40-42]. This questionnaire contains 9 items, each of which covers 1 DSM-IV criterion of MDD [39]. Questions are answered on a scale from 0 (not at all) to 3 (nearly every day), as experienced during the prior 2-week period. Sum scores indicate both the presence and the severity of depressive symptoms: none (0-4), mild (5-9), moderate (10-14), moderately severe (15-19), and severe depressive (20-27) symptoms [40,41]. The last item of the PHQ-9 evaluates suicidal ideation (ie, passive thoughts of death or self-injury). The researchers of E-COMPARED did not actively monitor or respond to reports of suicidal ideation, as this was not a stand-alone online treatment service. The MHC professional providing the therapy was considered well qualified and trained to identify and address suicide risk in their patients.

\section{Smartphone-Based EMA of Pleasant Activities, Pleasure, and Mood}

Information about activities, that is, the level of engagement in pleasant activities, on the day of reporting was gathered through the following question: "To what extent did you accomplish pleasant activities today?" The question was answered on a visual analogue scale (VAS) that ranged from 1 to 10 , with 1 precision digit after the decimal point and higher scores indicating more engagement in pleasant activities. Pleasure, that is, the subjective appraisal of the pleasure experienced through these activities, on the day of reporting was measured through the question "How much did you enjoy activities today?" This question was answered on a VAS scale that ranged from 1 to 10, with 1 precision digit after the decimal point and higher scores indicating greater pleasure experienced. Lastly, information pertaining to the patients' current mood was collected through the following question: "How is your mood right now?" This question was answered on a VAS scale that ranged from 1 (worst) to 10 (best), with 1 precision digital after the decimal point. Higher scores thus indicated more engagement in pleasant activities, greater pleasure experienced, and better mood on the day of reporting.

The EMA protocol varied over the course of the treatment. During both the first and the last 7 days of treatment, patients were prompted to rate their mood on 3 separate occasions each day (around 10:00 AM, 8:00 PM, and a random time between 10:00 AM and 10:00 PM). During these specific weeks, at the 8:00 PM prompt, patients were also invited to report on their level of pleasant activities and experienced pleasure. From the second week until the last week of treatment, patients were prompted to rate their mood once a day at a random time between 10:00 AM and 10:00 PM. Moreover, on 1 random evening (8:00 PM) each week, patients were also invited to rate their engagement in pleasant activities, experienced pleasure, and mood. Although patients were instructed to complete the questions as quickly as possible, they were given a time frame of $60 \mathrm{~min}$. Additionally, patients were also free to report their mood at any time other than the fixed prompts. The EMA protocol varied over the course of treatment, as the EMA component was used for supportive means in the treatment. The first week was for patients to get used to the EMA application, but it was considered unrealistic to expect from patients in a clinical setting to complete a full diary every day throughout the course of treatment $[35,36]$.

For this study, weekly averages of the EMA reports were calculated in order to ensure that all patients had an equal number of measurement points. The weekly averages of the 3 questions were calculated over a 3-month period, which resulted in 12 weekly EMA reports of activity, pleasure, and mood for each patient, as 3 months was deemed an appropriate timespan for examining the process of change, given the average length of bCBT protocols [36].

\section{Statistical Analysis}

Descriptive statistics were calculated for both baseline demographics and clinical characteristics. To answer the main research questions, linear mixed model (LMM) and random-intercept cross-lagged panel model (RI-CLPM) analyses were conducted. First, we performed multiple imputation (MI, $\mathrm{m}=100$ ) to impute the missing EMA weekly reports. In the data set, $18 \%, 54 \%$, and $54 \%$ of the 12 -week reports were missing for mood, pleasure, and activity, respectively. Full information maximum likelihood (FIML) and MI are 2 types of techniques that are considered best for handling missing data $[43,44]$. When conducting an RI-CLPM in RStudio, the default setting to handle missing observations is FIML [45]. We, however, chose to apply MI since this technique allows for a more convenient way to incorporate auxiliary variables in the model when running an RI-CLPM in RStudio. Auxiliary variables are additional covariates that are included in the model next to the variables ultimately analyzed in the final analysis. It is argued that adding auxiliary variables can substantially improve the handling of missing data [46]. 
We performed MI using the Amelia II-R-package (version 1.7.6) [47] as this allows for MI of time series data. The package uses a bootstrap-based expectation-maximization bootstrapping algorithm to impute missing observations. It uses all of the information present in the data set, allows for previously known information to be incorporated into the imputation model, and provides diagnostics of the model [47]. In this study, the patient ID was included as a fixed effect. Time was considered by including leads (previous measurement [t-1]) and lags (next measurement $[\mathrm{t}+1]$ ) into the imputation model. Previously known information was included by setting a logical bound between 1 and 10, as this was the answer range for the EMA questions. Auxiliary variables included gender, age, educational level, comorbid DSM-IV diagnoses at baseline, PHQ-9 at baseline, PHQ-9 after 3 months, and antidepressant usage at baseline. Diagnostics of the imputation model were checked by examining overimputation diagnostic plots. Please see Multimedia Appendix 1 [48-54] for more information about both missing data and the MI procedure.
Next, the development of engaging in activities, pleasure experienced, and mood state over the duration of the treatment was examined with 3 separate LMMs. Weekly averages of the EMA reports were added as dependent variables. To test our main research questions, unconstrained RI-CLPMs were estimated. Again, the weekly averages of the EMA reports were analyzed. The RI-CLPM, as proposed by Hamaker, Kuiper, and Grasman [55], is an extension of the traditional CLPM [56]. The CLPM is an expedient method for describing cross-lagged associations between variables [56]. However, 1 key drawback of the traditional CLPM is that within-person effects cannot be extracted; this is problematic, given that these effects reflect the intraindividual processes that are needed in order to be able to draw conclusions about how changes over time in one variable are linked to changes over time in another variable, with respect to the same person. The RI-CLPM decomposes the between- and within-person effects. Figure 2 provides a visual representation of an RI-CLPM.

Figure 2. RI-CLPM for 4 measurement points. $\alpha$ and $\zeta$ are autoregressive regression coefficients; $\gamma$ and $\delta$ are same-week (residual) covariances, $\beta$ and $\varepsilon$ are cross-lagged regression coefficients, and $\eta$ is between-person correlation. Based on Hamaker, Kuiper, and Grasman [55]. RI: random intercept; RI-CLPM: random-intercept cross-lagged panel model.

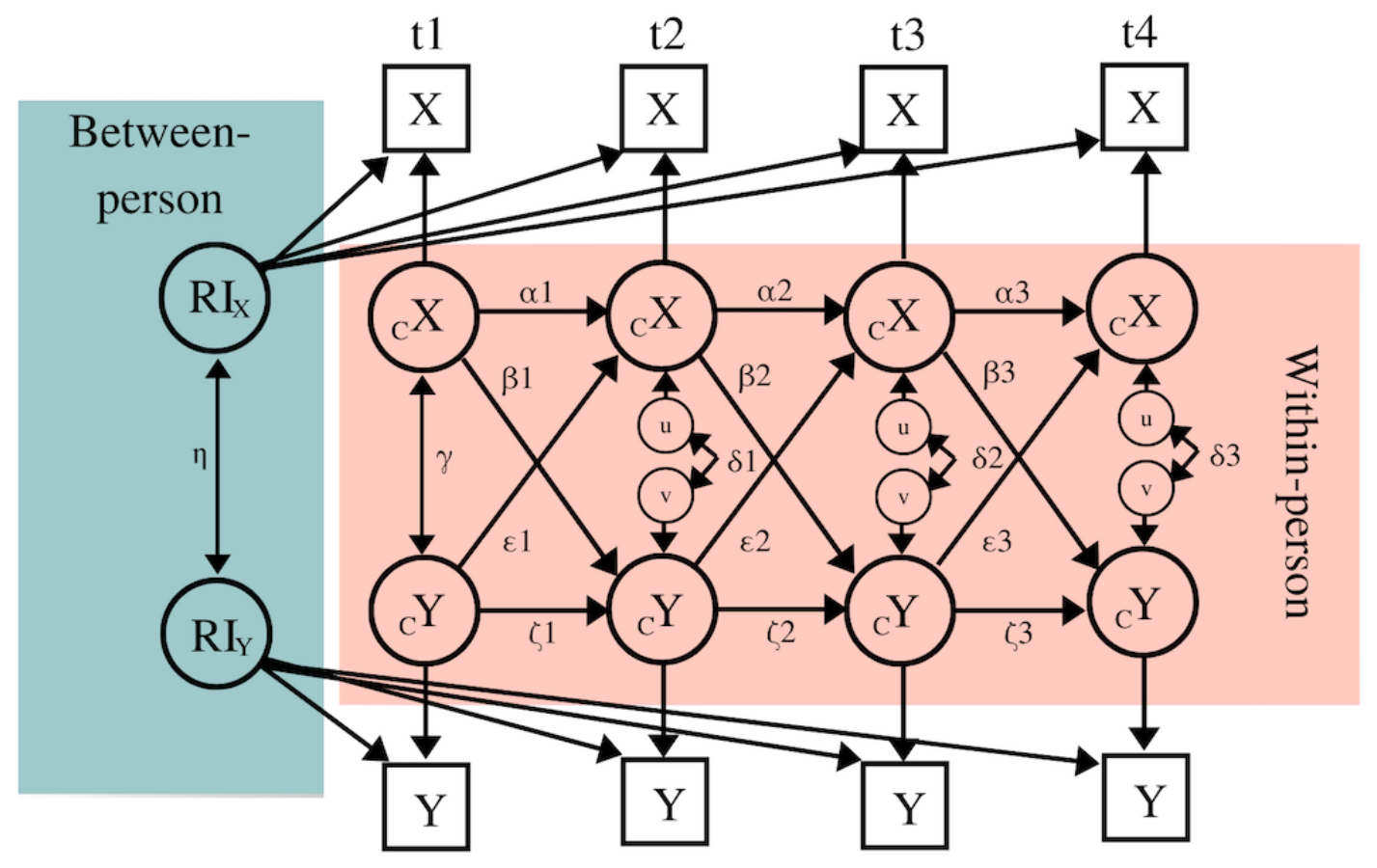

The RI-CLPM involves:

- $\quad$ Autoregression coefficients $(\alpha$ and $\zeta)$, which represent the carry-over effect (eg, $\mathrm{X}_{\mathrm{t}}$ on $\mathrm{X}_{t+1}$ ). A negative coefficient indicates that if the score for $\mathrm{X}$ in one week is above the overall mean, then the score for $\mathrm{X}$ in the following week is likely to be below the overall mean, and vice versa. Conversely, a positive coefficient indicates that a higher or lower score for $\mathrm{X}$ in one week corresponds to a higher or lower score for $\mathrm{X}$ in the following week.

- The cross-lagged coefficients $(\beta$ and $\varepsilon$ ) indicate the extent to which 2 variables influence each other. The coefficients show the extent to which any deviation in variable $\mathrm{X}$ in one week is related to any deviation in variable $\mathrm{Y}$ in the following week, when controlled for the autoregression of Y.

- The covariance $(\gamma)$ indicates the association between $X$ and $\mathrm{Y}$ in the first week, while the same-week residual covariances $(\delta)$ indicate the covariant change, that is, the extent to which a within-person change in $\mathrm{X}$ is associated with a within-person change in $\mathrm{Y}$ in the same week.

- The association between the random intercepts (RIs; $\eta$ ), which reflects the between-person effects over the course of the treatment.

The model fit of the RI-CLPMs was evaluated with (1) the $P$ value of the $\chi^{2}$ test statistic, (2) the standardized root-mean-square residual (SRMR), (3) the standardized 
root-mean-square error of approximation (RMSEA) [57], and (4) the comparative fit index (CFI) [58]. For an RI-CPLM to fit the data well, (1) the $\chi^{2}$ test statistic must be statistically nonsignificant $(P>.05)$, (2) the SRMR must be $\leq 0.08$, (3) the RMSEA must be $\leq 0.05$, and (4) the CFI must be $\geq 0.95$ [57-60].

All analyses were performed in RStudio ( $\mathrm{R}$ version 4.0.2.). The mitml-R-package was used to prepare the MI data sets for pooled analyses [61]. The LMMs were conducted using the nlme-R-package [62]. For the RI-CLPM, we derived our model syntax from the (basic) model syntax provided by Mulder and Hamaker [45]. To run the model syntax on the MI data sets, we needed the semTools-R-package [63]; this package provided the lavaan interface for the MI data sets $[63,64]$. Rubin's rules were applied to pool the results across the MI data sets [65]. $P<.05$ was considered statistically significant. A more detailed description of the RI-CLPM [55,66], the model syntax for the RI-CLPM [45], the Amelia II, lavaan, mitml, nmle, and semTools packages [47,61-64,67] and Rubin's rules [65] can be found elsewhere.

\section{Results}

\section{Sample Characteristics}

Table 2 shows the baseline demographics and clinical characteristics of the analytic sample. The sample comprised 240 participants, $66 \%$ (158/240) of which were female. The mean age was 37.3 years (SD 13.2), while 9\% (21/240), $34 \%$ $(80 / 240)$, and $58 \%(139 / 240)$ of the patients studied at an elementary, secondary, and higher educational level, respectively. One or more comorbid DSM-IV diagnoses were reported by 59\% (142/240) of the patients, while 30\% (71/240) were currently using antidepressant medication. Finally, 13\% (31/240) of the patients reported mild, 35\% (84/240) reported moderate, $30 \%$ (72/240) reported moderately severe, and $22 \%$ (53/240) reported severe depressive symptoms at baseline.

The analyses that were conducted to examine potential selection bias demonstrated that there was no difference in terms of demographics and clinical characteristics between the E-COMPARED patients who were randomly allocated to receive bCBT but did not meet the study's inclusion criteria (see the Methods section) $(n=476-240=226)$ and patients who were ultimately included in the study $(\mathrm{N}=240)$.

Table 2. Baseline demographics and clinical characteristics of the study sample $(\mathrm{N}=240)$.

\begin{tabular}{|c|c|}
\hline Characteristic & Patients, N (\%) \\
\hline \multicolumn{2}{|l|}{ Gender } \\
\hline Female & $158(66)$ \\
\hline Male & $82(34)$ \\
\hline \multicolumn{2}{|l|}{ Educational level } \\
\hline Elementary & $21(9)$ \\
\hline Secondary & $80(33)$ \\
\hline Higher & $139(58)$ \\
\hline \multicolumn{2}{|c|}{ Comorbid DSM-IV $^{\mathrm{a}}$ diagnoses $^{\mathrm{b}}$} \\
\hline 0 & $98(41)$ \\
\hline 1 & $77(32)$ \\
\hline 2 or more & $65(27)$ \\
\hline \multicolumn{2}{|l|}{ Antidepressant use } \\
\hline No & $169(70)$ \\
\hline Yes & $71(30)$ \\
\hline \multicolumn{2}{|l|}{ PHQ-9 ${ }^{c}$} \\
\hline Mild & $31(13)$ \\
\hline Moderate & $84(35)$ \\
\hline Moderately severe & $72(30)$ \\
\hline Severe & $53(22)$ \\
\hline
\end{tabular}

${ }^{a}$ DSM-IV: Diagnostic and Statistical Manual of Mental Disorders, Fourth Edition.

${ }^{\mathrm{b}}$ Current comorbid diagnoses included the DSM-IV diagnoses of dysthymia, panic disorder with or without agoraphobia, agoraphobia, social phobia, generalized anxiety disorder, and posttraumatic stress disorder.

${ }^{\mathrm{c}}$ PHQ-9: Patient Health Questionnaire-9. 


\section{Development of Engagement in Pleasant Activities, Experiencing Pleasure, and Mood During the Course of the Treatment}

Over the course of a 12-week period, patients provided weekly EMA reports concerning their activities, pleasure experienced, and mood. Since missing data were imputed with MI techniques (see Multimedia Appendix I), 12 weekly EMA reports for all 3 variables were available for each patient. On average, engagement in activities $\left(\mathrm{B}=0.02\right.$, SE $\left.0.01, t_{849}=2.03, P=.04\right)$, pleasure experienced $\left(\mathrm{B}=0.03, \mathrm{SE} 0.01, t_{943}=2.95, P=.003\right)$, and $\operatorname{mood}\left(\mathrm{B}=0.04, \mathrm{SE} 0.01, t_{1350}=6.2, P<.001\right)$ of patients all increased slightly during the course of the treatment. Age, gender, depression severity, and antidepressant usage were not considered confounders in any of the analyses.

\section{Longitudinal Reciprocal Associations in the BA Circle}

\section{Reciprocal Associations Between Engaging in Pleasant Activities and Experiencing Pleasure}

Figure 3 shows a simplified model for the reciprocal associations between engaging in pleasant activities and experiencing pleasure. The fit indices indicate that the model fits well $\left(\chi_{217}^{2}=52.35, P=.99, \mathrm{SMSR}=0.05, \mathrm{RMSEA}=0.00, \mathrm{CFI}=1.00\right)$.

Figure 3. Simplified RI-CLPM engaging in pleasant activities and experiencing pleasure. The between-person double-headed arrow represents a correlation. Within-person double-headed arrows represent (residual) covariances; single-headed arrows display standardized regression coefficients. Light-gray arrows represent nonsignificant covariances/coefficients. ${ }^{*} P<.001, * * P=.01$, $* * * P=.02$. A: activity; P: pleasure; RI: Random Intercept; RI-CLPM: random-intercept cross-lagged panel model.

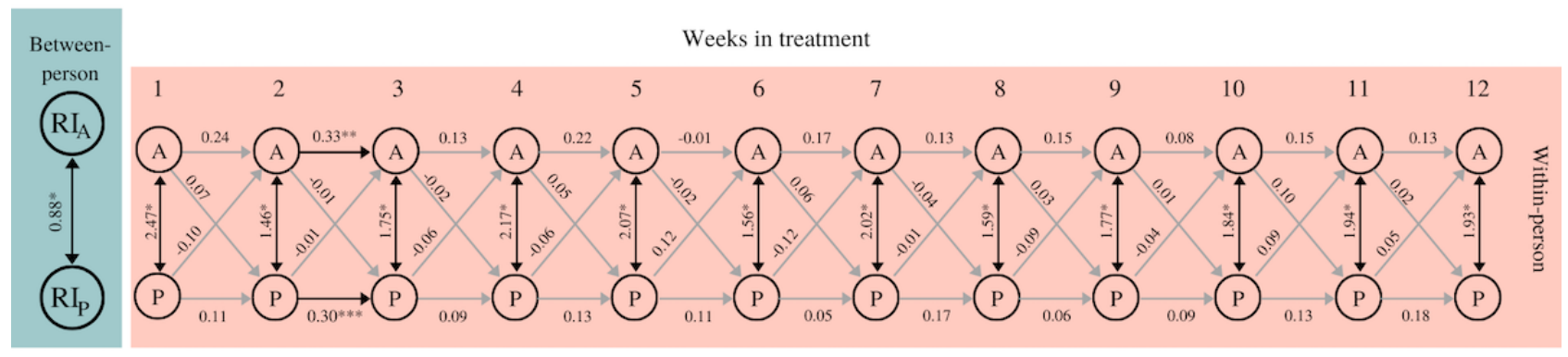

At the within-person level, none of the cross-lagged coefficients were statistically significant; engagement in activities was not predictive for experiencing pleasure, nor was the opposite the case. Regarding the autoregression coefficients, only 2 autoregressive paths were significant: the autoregressive path of engaging in activities between weeks 2 and $3(B=0.33, S E$ $0.12, P=.01)$ and the autoregressive path of experiencing pleasure between weeks 2 and $3(\mathrm{~B}=0.30$, SE $0.12, P=.02)$. The positive significant autoregressive pathway indicates that if the level of activities engaged in during week 2 was above the overall mean, then the level of activities engaged in during week 3 was also likely to be above the overall mean. This was also the case with respect to experiencing pleasure in weeks 2 and 3. Moreover, both the covariance between activity and pleasure in the first week (covariance $=2.47$ ) as well as the residual covariances from weeks 2 to 12 (range residual covariance $=1.46-2.07)$ were statistically significant (all $P<.001)$.
This means that increased engagement in pleasant activities was associated with an increase in the pleasure experienced in the same week.

At the between-person level, engaging in activities and experiencing pleasure were strongly correlated $(r=0.88, P<.001)$. This means that over the course of the treatment, patients who engaged more in pleasant activities also reported experiencing greater pleasure than those who engaged less in pleasant activities.

\section{Reciprocal Associations Between Experiencing Pleasure and Mood}

Figure 4 shows a simplified model for the reciprocal associations between experiencing pleasure and mood. The fit indices indicate that the model fits well $\left(\chi_{217}^{2}=91.67, P=.99\right.$, $\mathrm{SRMR}=0.08, \mathrm{RMSEA}=0.00, \mathrm{CFI}=1.00)$.

Figure 4. Simplified RI-CLPM experiencing pleasure and mood. The between-person double-headed arrow represents a correlation. Within-person double-headed arrows represent (residual) covariances; single-headed arrows display standardized regression coefficients. Light-gray arrows represent nonsignificant covariances/coefficients. ${ }^{*} P<.001$, $* * P=.01$. M: mood; P: pleasure; RI: Random Intercept; RI-CLPM: random-intercept cross-lagged panel model.

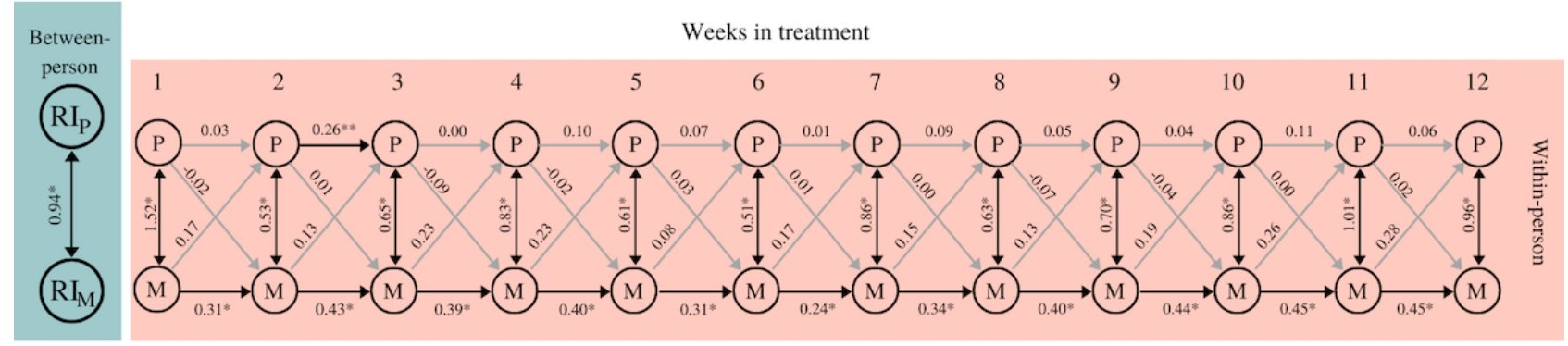


At the within-person level, none of the cross-lagged coefficients were statistically significant; the level of pleasure was not predictive for mood 1 week later, nor vice versa. With regard to the autoregression coefficients, the autoregressive path between weeks 2 and 3 was statistically significant with respect to experiencing pleasure $(\mathrm{B}=0.26$, SE $0.10, P=.01)$, while all the coefficients of mood appeared to be positive and significant $(\mathrm{B}=0.24-0.45, P<.001)$. This positive significant autoregressive pathway thus indicates that if the level of experiencing pleasure (or mood) in week t was above the overall mean, then the level of experiencing pleasure (or mood) in week $\mathrm{t}+1$ was also likely to be above the overall mean. Moreover, both the covariance between experiencing pleasure and mood in the first week (covariance $=1.52$ ) as well as the residual covariances from weeks 2 to 12 (range residual covariance=0.51-1.01) were statistically significant (all $P<.001$ ). This means that an increase in experienced pleasure was associated with an increase in mood in the same week.

At the between-person level, experiencing pleasure and mood were strongly correlated $(r=0.94, P<.001)$. This means that over the duration of the treatment, patients who experienced more pleasure also reported being in a better mood than patients who experienced less pleasure.

\section{Reciprocal Associations Between Mood and Engaging in Pleasant Activities}

Figure 5 shows a simplified model for the reciprocal associations between mood and engagement in pleasant activities. The fit indices indicate that the model fits well $\left(\chi_{217}^{2}=98.65, P=.99\right.$, $\mathrm{SRMR}=0.08, \mathrm{RMSEA}=0.00, \mathrm{CFI}=1.00$ ).

Figure 5. Simplified RI-CLPM mood and engaging in pleasant activities. The between-person double-headed arrow represents a correlation. Within-person double-headed arrows represent (residual) covariances; single-headed arrows display standardized regression coefficients. Light-gray arrows represent nonsignificant covariances/coefficients. $* P<.001$, $* * P=.02$. A: activity; M: mood; RI: Random Intercept; RI-CLPM: random-intercept cross-lagged panel model.

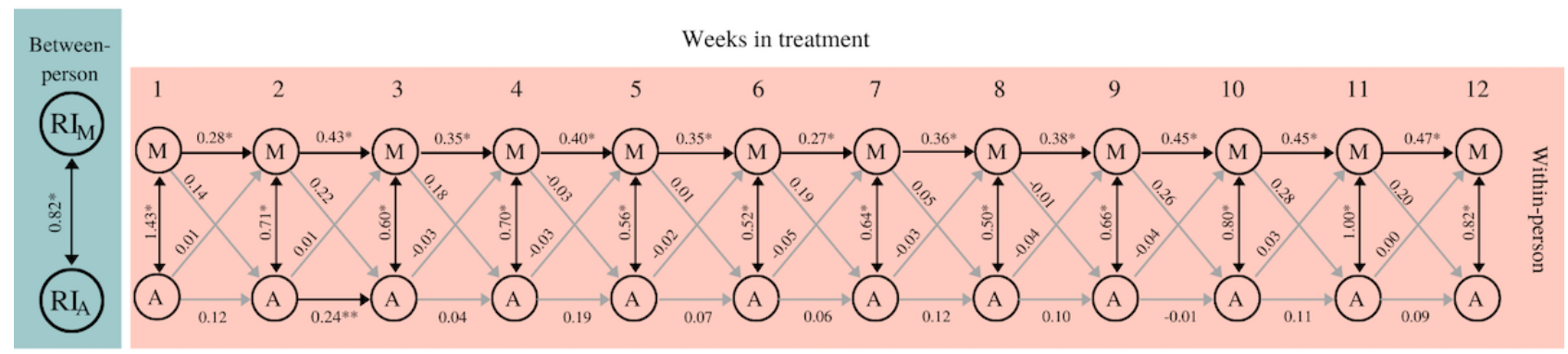

At the within-person level, none of the cross-lagged coefficients were statistically significant. Mood was not predictive of engagement in activities 1 week later, nor vice versa. With regard to the autoregression coefficients concerned, autoregressive paths for mood were found to be positive and significant $(\mathrm{B}=0.28-0.46, P<.001)$, while for engagement in activities, only the autoregressive path between weeks 2 and 3 $(\mathrm{B}=0.24, P=.02)$ was significant. Moreover, both the covariance between mood and engagement in activities in the first week (covariance $=1.43$ ) as well as the residual covariances from weeks 2 to 12 (range residual covariance=0.50-1.00) were statistically significant (all $P<.001$ ). This means that an increase in mood was associated with an increase in engagement in activities in the same week.

At the between-person level, there was a strong correlation between mood and engagement in activities $(r=0.82, P<.001)$. This means that over the course of the treatment, patients who experienced improved mood also reported higher levels of engagement in activities.

\section{Discussion}

\section{Principal Findings}

This study is a first attempt in examining the (temporal) associations between the 3 constructs of the theoretical framework of BA, which is grounded in Lewinsohn et al's $[14,15]$ theory of depression, among MDD patients during the course of bCBT. We found no evidence of a temporal

relationship between the 3 variables of interest: engagement in pleasant activities, experiencing pleasure, and mood. However, the results did reveal cross-sectional positive relationships between the 3 variables. Moreover, the levels of engagement in activities, pleasure, and mood increased slightly over the course of the treatment. In addition, mood appeared to be self-predictive over time, which was not the case for either engagement in activities or pleasure. Finally, over the course of the treatment, strong positive correlations between engagement in activities, pleasure, and mood were identified at the between-patient level.

\section{Comparison With Previous Literature}

First, we were interested in examining the temporal dimension of the theoretical framework of BA. In this respect, our findings did not reveal any temporal relationships between engagement in activities and the subsequent pleasure and mood experienced in the following weeks. This result was somewhat surprising as it is not in accordance with Lewinsohn et al's [14,15] theory of depression, which clearly posits a temporal relationship between engagement in activities and pleasure, pleasure and mood, and mood and activities (see Figure 1). This raises the question of whether the lack of temporal relationships in our study stems from the selected lag (weekly interval).

The selected lag was a consequence of the chosen sampling scheme in the treatment protocol. We could only use the data that were available, and the E-COMPARED study was not designed to investigate the theoretical framework of BA. To avoid false-negative or false-positive findings, it is vitally 
important to choose the right lag; however, this is difficult as the right lag cannot be standardized but, rather, is completely dependent on the research topic $[68,69]$. In the case of our study, it could well be that the temporal relationships would have been revealed if the time intervals between the subsequent measurements had been shorter (eg, daily rather than weekly EMA reports).

The question of whether the data were suitable for illustrating temporal relationships is further evoked by the fact that same-week relationships between the 3 examined variables were identified $[68,69]$. That is, in those weeks when a patient (1) engaged in more pleasant activities, they also reported an increase in pleasure; (2) experienced more pleasure, they also reported a better mood; and (3) reported a better mood, they also engaged in more pleasant activities. Although these within-patient associations are potentially in line with the theoretical framework of BA [14,15], since these same-week relationships are cross-sectional, it cannot be established whether there is causal dominance, equal reciprocal relationships, or cyclical relationships.

Moreover, the analyses highlighted between-patient effects; patients who engaged more in pleasant activities over the course of the treatment also reported experiencing more pleasure. Patients who experienced more pleasure also reported a better mood, while patients who reported a better mood also reported engaging in more activities over the course of the treatment. Although we were primarily interested in intrapatient processes and causal dominance cannot be established, the between-patient effects do complement the intrapatient effects, insofar as the positive relationship between the 3 variables of interest at the between-patient level does fit within the scope of the theoretical framework of BA $[14,15]$. To the best of our knowledge, this was the first (EMA) study to examine these effects over the course of treatment within a sample of adult MDD patients in routine $\mathrm{MHC}$; prior studies have either focused on comparing healthy controls with depressed persons, who are often recruited from the general population (eg, [70-72]), or investigated activities or pleasure as a mediator of pre- to postdepression severity change after depression treatment $[20,73]$.

\section{Limitations}

In addition to the question of whether the selected time lag was appropriate, the results should also be considered with a further limitation in mind, namely the proportion of missing weekly EMA reports. In particular, the adherence rates for the engagement in activity and pleasure reports were relatively poor (both $46 \%$ ). However, we contend that we were able to mitigate this problem by carrying out MI on the missing weekly EMA reports.

\section{Clinical Implications and Future Research}

This study addresses a noteworthy gap in the extant literature and, as such, can be regarded as constituting an important first step toward establishing evidence for the theoretical framework of BA among MDD patients. To the best of our knowledge, this represents the first study to investigate intrapatient processes of BA during the course of (b)CBT treatment. The results lend at least some empirical support for the underlying theoretical framework of a therapy that is regarded by the American Psychological Association [74] as 1 of the recommended treatments for adult depression. A next step would be to replicate the study across a different data set, possibly with smaller time intervals between the measurements. Moreover, in our study, we focused on whether we could find associations between the 3 key constructs that are part of the theoretical framework of BA for MDD. We do know that BA starts early in treatment and remains a recurring topic in the (f-t-f) sessions throughout the entire course of treatment. However, based on our data, we could not tease apart the influence of other intervention components from the influence of BA, as BA was part of CBT. Whether BA succeeds in activating MDD patients would be an interesting question that should be explored in future work.

\section{Conclusions}

The results of our study are partially in accordance with the theoretical framework of BA. The analyses demonstrated statistically significant cross-sectional relationships between levels of engagement in activity, pleasure experienced as a result of these activities, and mood. However, as we did not reveal any statistically significant temporal relationships, no conclusions could be drawn concerning possible causality. A shorter measurement interval (eg, daily rather than weekly EMA reports) might be more conducive to detecting potential underlying temporal pathways. Consequently, future research should use an EMA methodology to further investigate these temporal associations, based on theory and how the treatments are presented to patients.

\section{Acknowledgments}

The European Comparative Effectiveness Research on Internet-based Depression Treatment versus treatment-as-usual (E-COMPARED) project is funded by the European Commission FP7-Health-2013-Innovation-1 program (grant no. 603098-2).

\section{Conflicts of Interest}

DDE has served as a consultant to/on the scientific advisory boards of Sanofi, Novartis, Minddistrict, Lantern, Schoen Klinike, Ideamed and German health insurance companies (BARMER, Techniker Krankenkasse) and a number of federal chambers for psychotherapy. He is also stakeholder of the Institute for health training online (formerly GET.ON/ now HelloBetter), which aims to implement scientific findings related to digital health interventions into routine care. IT reports to have received fees for lectures/workshops in the e-mental-health context from training institutes for psychotherapists. All other authors declare that they have no conflicts of interest. 


\section{Multimedia Appendix 1}

Multiple imputation of missing data.

[DOC File, $30 \mathrm{~KB}-$ Multimedia Appendix 1]

\section{References}

1. Cuijpers P, Noma H, Karyotaki E, Vinkers CH, Cipriani A, Furukawa TA. A network meta-analysis of the effects of psychotherapies, pharmacotherapies and their combination in the treatment of adult depression. World Psychiatry 2020 Feb 10;19(1):92-107 [FREE Full text] [doi: 10.1002/wps.20701] [Medline: 31922679]

2. Munder T, Flückiger C, Leichsenring F, Abbass AA, Hilsenroth MJ, Luyten P, et al. Is psychotherapy effective? A re-analysis of treatments for depression. Epidemiol Psychiatr Sci 2018 Jul 30;28(03):268-274. [doi: 10.1017/s2045796018000355]

3. Cuijpers P, Karyotaki E, Eckshtain D, Ng MY, Corteselli KA, Noma H, et al. Psychotherapy for depression across different age groups: a systematic review and meta-analysis. JAMA Psychiatry 2020 Jul 01;77(7):694-702 [REEE Full text] [doi: 10.1001/jamapsychiatry.2020.0164] [Medline: $\underline{\text { 32186668] }}$

4. Cuijpers P, Oud M, Karyotaki E, Noma H, Quero S, Cipriani A, et al. Psychologic treatment of depression compared with pharmacotherapy and combined treatment in primary care: a network meta-analysis. Ann Fam Med 2021 May 10;19(3):262-270 [FREE Full text] [doi: 10.1370/afm.2676] [Medline: 34180847]

5. Cuijpers P, Reijnders M, Huibers MJ. The role of common factors in psychotherapy outcomes. Annu Rev Clin Psychol 2019 May 07;15(1):207-231. [doi: 10.1146/annurev-clinpsy-050718-095424] [Medline: 30550721]

6. Kazdin AE. Mediators and mechanisms of change in psychotherapy research. Annu Rev Clin Psychol 2007 Apr;3:1-27. [doi: 10.1146/annurev.clinpsy.3.022806.091432] [Medline: 17716046 ]

7. Kazdin AE. Understanding how and why psychotherapy leads to change. Psychother Res 2009 Jul 22;19(4-5):418-428. [doi: 10.1080/10503300802448899] [Medline: 19034715$]$

8. Domhardt M, Steubl L, Boettcher J, Buntrock C, Karyotaki E, Ebert DD, et al. Mediators and mechanisms of change in internet- and mobile-based interventions for depression: a systematic review. Clin Psychol Rev 2021 Feb;83:101953. [doi: 10.1016/j.cpr.2020.101953] [Medline: 33422841]

9. Moldovan R, Pintea S. Mechanisms of change in psychotherapy: methodological and statistical considerations. Cogn Brain Behav An Interdiscipl J 2015 Dec;4:299-311.

10. Lemmens LH, Müller VNLS, Arntz A, Huibers MJ. Mechanisms of change in psychotherapy for depression: an empirical update and evaluation of research aimed at identifying psychological mediators. Clin Psychol Rev 2016 Dec;50:95-107. [doi: 10.1016/j.cpr.2016.09.004] [Medline: 27770716]

11. Dimidjian S, Barrera M, Martell C, Muñoz RF, Lewinsohn PM. The origins and current status of behavioral activation treatments for depression. Annu Rev Clin Psychol 2011 Apr 27;7(1):1-38. [doi: 10.1146/annurev-clinpsy-032210-104535] [Medline: 21275642]

12. Uphoff E, Ekers D, Robertson L, Dawson S, Sanger E, South E, et al. Behavioural activation therapy for depression in adults. Cochrane Database Syst Rev 2020 Jul 06;7:CD013305 [FREE Full text] [doi: 10.1002/14651858.CD013305.pub2] [Medline: $\underline{\text { 32628293] }}$

13. Stein AT, Carl E, Cuijpers P, Karyotaki E, Smits JAJ. Looking beyond depression: a meta-analysis of the effect of behavioral activation on depression, anxiety, and activation. Psychol Med 2020 Mar 06;51(9):1491-1504. [doi: $10.1017 / \mathrm{s} 0033291720000239]$

14. Lewinsohn P. A behavioral approach to depression. In: Freedman R, Katz M, editors. The Psychology of Depression. Oxford: Wiley; 1974:157-174.

15. Lewinsohn P, Munoz R, Youngren M, Zeiss A. Control Your Depression. 2nd ed. New York: Prentice-Hall; 1986:978-0671762421.

16. Cuijpers P, van Straten A, Warmerdam L. Behavioral activation treatments of depression: a meta-analysis. Clin Psychol Rev 2007 Apr;27(3):318-326. [doi: 10.1016/J.CPR.2006.11.001]

17. Keijers G, van MA, Verbraak M, Hoogduin K, Emmelkamp P. Protocollaire Behandelingen Voor Volwassenen Met Psychische Klachten, Deel. Amsterdam: Boom; 2017.

18. Nagy GA, Cernasov P, Pisoni A, Walsh E, Dichter GS, Smoski MJ. Reward network modulation as a mechanism of change in behavioral activation. Behav Modif 2020 Mar 13;44(2):186-213 [FREE Full text] [doi: 10.1177/0145445518805682] [Medline: $\underline{30317863}$ ]

19. Forbes CN. New directions in behavioral activation: using findings from basic science and translational neuroscience to inform the exploration of potential mechanisms of change. Clin Psychol Rev 2020 Jul;79:101860. [doi:

10.1016/j.cpr.2020.101860] [Medline: 32413734]

20. Janssen N, Hendriks G, Baranelli C, Lucassen P, Oude Voshaar R, Spijker J, et al. How does behavioural activation work? A systematic review of the evidence on potential mediators. Psychother Psychosom 2021 Sep 8;90(2):85-93 [FREE Full text] [doi: $\underline{10.1159 / 000509820}$ ] [Medline: $\underline{32898847}$ ]

21. Bandura A. The self system in reciprocal determinism. Am Psychol 1978;33(4):344-358. [doi: 10.1037/0003-066x.33.4.344] 
22. Hoet AC, Burgin CJ, Eddington KM, Silvia PJ. Reports of therapy skill use and their efficacy in daily life in the short-term treatment of depression. Cogn Ther Res 2017 Apr 13;42(2):184-192. [doi: 10.1007/s10608-017-9852-y]

23. Stone A, Shiffman S. Ecological momentary assessment (EMA) in behavioral medicine. Ann Behav Med 1994;16(3):199-202. [doi: 10.1093/abm/16.3.199]

24. Shiffman S, Stone AA, Hufford MR. Ecological momentary assessment. Annu Rev Clin Psychol 2008 Apr;4(1):1-32. [doi: 10.1146/annurev.clinpsy.3.022806.091415] [Medline: 18509902]

25. Trull TJ, Ebner-Priemer UW. Ambulatory assessment in psychopathology research: a review of recommended reporting guidelines and current practices. J Abnorm Psychol 2020 Jan;129(1):56-63. [doi: 10.1037/abn0000473] [Medline: 31868388]

26. Wenze SJ, Miller IW. Use of ecological momentary assessment in mood disorders research. Clin Psychol Rev 2010 Aug;30(6):794-804. [doi: 10.1016/j.cpr.2010.06.007] [Medline: 20619520]

27. Colombo D, Fernández-Álvarez J, Patané A, Semonella M, Kwiatkowska M, García-Palacios A, et al. Current state and future directions of technology-based ecological momentary assessment and intervention for major depressive disorder: a systematic review. J Clin Med 2019 Apr 05;8(4):465 [FREE Full text] [doi: 10.3390/jcm8040465] [Medline: 30959828]

28. van Genugten CR, Schuurmans J, Lamers F, Riese H, Penninx BWJH, Schoevers RA, et al. Experienced burden of and adherence to smartphone-based ecological momentary assessment in persons with affective disorders. J Clin Med 2020 Jan 23;9(2):322 [FREE Full text] [doi: 10.3390/jcm9020322] [Medline: 31979340]

29. Ellison WD, Trahan AC, Pinzon JC, Gillespie ME, Simmons LM, King KY. For whom, and for what, is experience sampling more accurate than retrospective report? Pers Individ Dif 2020 Sep;163:110071. [doi: 10.1016/j.paid.2020.110071]

30. Solhan MB, Trull TJ, Jahng S, Wood PK. Clinical assessment of affective instability: comparing EMA indices, questionnaire reports, and retrospective recall. Psychol Assess 2009 Sep;21(3):425-436 [FREE Full text] [doi: 10.1037/a0016869] [Medline: 19719353]

31. Claúdio V, Garcez Aurélio J, Machado PPP. Autobiographical memories in major depressive disorder. Clin Psychol Psychother 2012 Sep 12;19(5):375-389. [doi: 10.1002/cpp.751] [Medline: 21567654]

32. Dalgleish T, Werner-Seidler A. Disruptions in autobiographical memory processing in depression and the emergence of memory therapeutics. Trends Cogn Sci 2014 Nov;18(11):596-604. [doi: 10.1016/j.tics.2014.06.010] [Medline: 25060510]

33. Köhler CA, Carvalho A, Alves G, McIntyre R, Hyphantis T, Cammarota M. Autobiographical memory disturbances in depression: a novel therapeutic target? Neural Plast 2015;2015:759139 [FREE Full text] [doi: 10.1155/2015/759139] [Medline: 26380121]

34. Riper H, van BW, Kooistra L, de WJ, Donker T. Preventie \& EMental-Health - Prevention \& EMental Health. Amsterdam, the Netherlands: Vrije Universiteit commissioned by ZonMw; 2013:1-132.

35. Kemmeren LL, van Schaik A, Smit JH, Ruwaard J, Rocha A, Henriques M, et al. Unraveling the black box: exploring usage patterns of a blended treatment for depression in a multicenter study. JMIR Ment Health 2019 Jul 25;6(7):e12707 [FREE Full text] [doi: 10.2196/12707] [Medline: $\underline{\text { 31344670] }}$

36. Kleiboer A, Smit J, Bosmans J, Ruwaard J, Andersson G, Topooco N, et al. European COMPARative Effectiveness research on blended Depression treatment versus treatment-as-usual (E-COMPARED): study protocol for a randomized controlled, non-inferiority trial in eight European countries. Trials 2016 Aug 03;17(1):387 [FREE Full text] [doi: 10.1186/s13063-016-1511-1] [Medline: 27488181]

37. Kooistra L. Blended CBT for Depression. PhD thesis. Amsterdam, the Netherlands: Vrije Universiteit; 2020.

38. Sheehan DV, Lecrubier Y, Sheehan KH, Amorim P, Janavs J, Weiller E, et al. The Mini-International Neuropsychiatric Interview (M.I.N.I.): the development and validation of a structured diagnostic psychiatric interview for DSM-IV and ICD-10. J Clin Psychiatry 1998;59 Suppl 20:22-33;quiz 34. [Medline: 9881538]

39. American PA. Diagnostic and Statistical Manual of Mental Disorders, 4th Ed. Washington, DC: American Psychiatric Association; 1994.

40. Kroenke K, Spitzer RL, Williams JBW. The PHQ-9: validity of a brief depression severity measure. J Gen Intern Med 2001 Sep;16(9):606-613 [FREE Full text] [doi: 10.1046/j.1525-1497.2001.016009606.x] [Medline: 11556941$]$

41. Kroenke K, Spitzer RL, Williams JBW, Löwe B. The Patient Health Questionnaire Somatic, Anxiety, and Depressive Symptom Scales: a systematic review. Gen Hosp Psychiatry 2010 Jul;32(4):345-359. [doi: 10.1016/j.genhosppsych.2010.03.006] [Medline: 20633738]

42. van Ballegooijen W, Riper H, Cuijpers P, van Oppen P, Smit JH. Validation of online psychometric instruments for common mental health disorders: a systematic review. BMC Psychiatry 2016 Feb 25;16(1):45 [FREE Full text] [doi: 10.1186/s12888-016-0735-7] [Medline: 26915661]

43. Schafer JL, Graham JW. Missing data: our view of the state of the art. Psychol Methods 2002;7(2):147-177. [doi: 10.1037/1082-989x.7.2.147]

44. Lee T, Shi D. A comparison of full information maximum likelihood and multiple imputation in structural equation modeling with missing data. Psychol Methods 2021 Aug 28;26(4):466-485. [doi: 10.1037/met0000381] [Medline: 33507765]

45. Mulder J, Hamaker E. The RI-CLPM \& Extensions. URL: https://jeroendmulder.github.io/RI-CLPM/lavaan.html [accessed 2021-04-30]

46. Graham JW. Adding missing-data-relevant variables to FIML-based structural equation models. Struct Equ Model 2003 Jan;10(1):80-100. [doi: 10.1207/s15328007sem1001 4] 
47. Honaker J, King G, Blackwell M. AMELIA II: a program for missing data. J Stat Soft 2011 Dec 11;45(7):1-45. [doi: 10.18637/jss.v045.i07]

48. Pedersen A, Mikkelsen E, Cronin-Fenton D, Kristensen N, Pham TM, Pedersen L, et al. Missing data and multiple imputation in clinical epidemiological research. CLEP 2017 Mar; Volume 9:157-166. [doi: 10.2147/clep.s129785]

49. Lee JH, Huber JC. Evaluation of multiple imputation with large proportions of missing data: how much is too much? 2011 Presented at: United Kingdom Stata Users' Group Meetings; 2011; United Kingdom.

50. van Buuren S. Flexible Imputation of Missing Data. 2nd edition. Boca Raton, FL: Taylor \& Francis; 2018. [doi: $10.1201 / 9780429492259]$

51. Rubin DB. Inference and missing data. Biometrika 1976 Dec;63(3):581-592. [doi: 10.2307/2335739]

52. Carter R. Solutions for missing data in structural equation modeling. Res Pract Assess 2006;1:4-7.

53. Wolgast A, Schwinger M, Hahnel C, Stiensmeier-Pelster J. Handling missing data in structural equation models in R. A replication study for applied researchers. EJREP 2017 Dec 08;15(41):5-47. [doi: 10.25115/ejrep.41.16125]

54. Little RJA. A test of missing completely at random for multivariate data with missing values. J Am Stat Assoc 1988 Dec;83(404):1198-1202. [doi: 10.2307/2290157]

55. Hamaker EL, Kuiper RM, Grasman RPPP. A critique of the cross-lagged panel model. Psychol Methods 2015 Mar;20(1):102-116. [doi: 10.1037/a0038889] [Medline: 25822208]

56. Kearney M. Cross lagged panel analysis. In: Allen M, editor. The SAGE Encyclopedia of Communication Research Methods. Thousand Oaks, CA: Sage; 2017:312-314.

57. Steiger JH. Structural model evaluation and modification: an interval estimation approach. Multivariate Behav Res 1990 Apr 01;25(2):173-180. [doi: 10.1207/s15327906mbr2502 4] [Medline: 26794479]

58. Bentler PM. Comparative fit indexes in structural models. Psychol Bull 1990 Mar;107(2):238-246. [doi: 10.1037/0033-2909.107.2.238] [Medline: 2320703]

59. Hu L, Bentler PM. Cutoff criteria for fit indexes in covariance structure analysis: conventional criteria versus new alternatives. Struct Equ Model 1999 Jan;6(1):1-55. [doi: 10.1080/10705519909540118]

60. Iacobucci D. Structural equations modeling: fit indices, sample size, and advanced topics. J Consum Psychol 2010 Jan;20(1):90-98. [doi: 10.1016/j.jcps.2009.09.003] [Medline: 25904163]

61. Grund S, Robitzsch A, Luedtke O. Tools for Multiple Imputation in Multilevel Modeling. URL: https://cran.r-project.org/ web/packages/mitml/mitml.pdf [accessed 2021-02-02]

62. Pinheiro J, Bates D, DebRoy S, Sarker D, R Core Team. nlme: Linear and Nonlinear Mixed Effects Models (version 3. URL: https://CRAN.R-project.org/package=nlme [accessed 2021-01-30]

63. Jorgensen T, Pornprasertmanit S, Schoemann A, Rosseel Y, Miller P, Quick C, et al. semTools: Useful Tools for Structural Equation Modeling. 2021. URL: https://CRAN.R-project.org/package=semTools [accessed 2021-04-30]

64. Rosseel Y. lavaan: an R package for structural equation modeling. J Stat Soft 2012 May 24;48(2):1-36. [doi: 10.18637/jss.v048.i02]

65. Rubin DB. Multiple Imputation for Nonresponse in Surveys. Hoboken, NJ: John Wiley \& Sons; 1987.

66. Mulder JD, Hamaker EL. Three extensions of the random intercept cross-lagged panel model. Struct Equ Model 2020 Aug 11;28(4):638-648. [doi: 10.1080/10705511.2020.1784738]

67. Zhang Z. Multiple imputation for time series data with Amelia package. Ann Transl Med 2016 Feb;4(3):56 [REE Full text] [doi: 10.3978/j.issn.2305-5839.2015.12.60] [Medline: 26904578]

68. Vaisey S, Miles A. What you can — and can't_-do with three-wave panel data. Sociol Methods Res 2016 Jul 08;46(1):44-67. [doi: $10.1177 / 0049124114547769]$

69. Allison P. Getting the Lags Right. URL: https://statisticalhorizons.com/getting-the-lags-right [accessed 2021-05-10]

70. Lamers F, Swendsen J, Cui L, Husky M, Johns J, Zipunnikov V, et al. Mood reactivity and affective dynamics in mood and anxiety disorders. J Abnorm Psychol 2018 Oct;127(7):659-669. [doi: 10.1037/abn0000378] [Medline: 30335438]

71. Heininga VE, Dejonckheere E, Houben M, Obbels J, Sienaert P, Leroy B, et al. The dynamical signature of anhedonia in major depressive disorder: positive emotion dynamics, reactivity, and recovery. BMC Psychiatry 2019 Feb 08;19(1):59 [FREE Full text] [doi: 10.1186/s12888-018-1983-5] [Medline: 30736751]

72. Thompson RJ, Mata J, Jaeggi SM, Buschkuehl M, Jonides J, Gotlib IH. The everyday emotional experience of adults with major depressive disorder: examining emotional instability, inertia, and reactivity. J Abnorm Psychol 2012 Nov;121(4):819-829 [FREE Full text] [doi: 10.1037/a0027978] [Medline: 22708886]

73. Hershenberg R, Paulson D, Gros DF, Acierno R. Does amount and type of activity matter in behavioral activation? A preliminary investigation of the relationship between pleasant, functional, and social activities and outcome. Behav Cogn Psychother 2014 Mar 13;43(4):396-411. [doi: 10.1017/s1352465813001185]

74. American Psychological Association. APA Guideline for the Treatment of Depression. 2019. URL: https://www.apa.org/ depression-guideline/guideline.pdf [accessed 2021-05-10]

\section{Abbreviations}

BA: behavioral activation 
bCBT: blended cognitive behavioral therapy

CFI: comparative fit index

DSM-IV: Diagnostic and Statistical Manual of Mental Disorders, Fourth Edition

E-COMPARED: European Comparative Effectiveness Research on Internet-based Depression Treatment versus treatment-as-usual

EMA: ecological momentary assessment

FIML: full information maximum likelihood

f-t-f: face-to-face

LMM: linear mixed model

MDD: major depressive disorder

MHC: mental health care

MI: multiple imputation

PHQ-9: Patient Health Questionnaire-9

RI: random intercept

RI-CLPM: random-intercept cross-lagged panel model

RMSEA: root-mean-square error of approximation

SRMR: standardized root-mean-square residual

TAU: treatment-as-usual

VAS: visual analogue scale

\author{
Edited by G Eysenbach; submitted 19.07.21; peer-reviewed by D Fulford; comments to author 10.08.21; revised version received \\ 06.10.21; accepted 28.10.21; published 06.12.21 \\ Please cite as: \\ van Genugten CR, Schuurmans J, Hoogendoorn AW, Araya R, Andersson G, Baños R, Botella C, Cerga Pashoja A, Cieslak R, Ebert \\ DD, García-Palacios A, Hazo JB, Herrero R, Holtzmann J, Kemmeren L, Kleiboer A, Krieger T, Smoktunowicz E, Titzler I, Topooco \\ $N$, Urech A, Smit JH, Riper $H$ \\ Examining the Theoretical Framework of Behavioral Activation for Major Depressive Disorder: Smartphone-Based Ecological \\ Momentary Assessment Study \\ JMIR Ment Health 2021;8(12):e32007 \\ URL: https://mental.jmir.org/2021/12/e32007 \\ doi: $\underline{10.2196 / 32007}$ \\ PMID: $\underline{34874888}$
}

(C) Claire Rosalie van Genugten, Josien Schuurmans, Adriaan W Hoogendoorn, Ricardo Araya, Gerhard Andersson, Rosa Baños, Cristina Botella, Arlinda Cerga Pashoja, Roman Cieslak, David Daniel Ebert, Azucena García-Palacios, Jean-Baptiste Hazo, Rocío Herrero, Jérôme Holtzmann, Lise Kemmeren, Annet Kleiboer, Tobias Krieger, Ewelina Smoktunowicz, Ingrid Titzler, Naira Topooco, Antoine Urech, Johannes H Smit, Heleen Riper. Originally published in JMIR Mental Health (https://mental.jmir.org), 06.12.2021. This is an open-access article distributed under the terms of the Creative Commons Attribution License (https://creativecommons.org/licenses/by/4.0/), which permits unrestricted use, distribution, and reproduction in any medium, provided the original work, first published in JMIR Mental Health, is properly cited. The complete bibliographic information, a link to the original publication on https://mental.jmir.org/, as well as this copyright and license information must be included. 